\title{
Joint TDD Backhaul and Access Optimization in Dense Small Cell Networks
}

\author{
Mehrdad Shariat, Emmanouil Pateromichelakis, Atta ul Quddus and Rahim Tafazolli
}

\begin{abstract}
This paper addresses the problem of joint backhaul and access links optimization in dense small cell networks with special focus on time division duplexing (TDD) mode of operation in backhaul and access links transmission. Here, we propose a framework for joint radio resource management where we systematically decompose the problem in backhaul and access links. To simplify the analysis, the procedure is tackled in two stages. At the first stage, the joint optimization problem is formulated for a point-to-point scenario where each small cell is simply associated to a single user. It is shown that the optimization can be decomposed into separate power and subchannel allocation in both backhaul and access links where a set of rate-balancing parameters in conjunction with duration of transmission governs the coupling across both links. Moreover, a novel algorithm is proposed based on grouping the cells to achieve rate-balancing in different small cells. Next in the second stage, the problem is generalized for multi access small cells. Here, each small cell is associated to multiple users to provide the service. The optimization is similarly decomposed into separate sub-channel and power allocation by employing auxiliary slicing variables. It is shown that similar algorithms as previous stage are applicable by slight change with the aid of slicing variables. Additionally, for the special case of line-of-sight backhaul links, simplified expressions for sub-channel and power allocation are presented. The developed concepts are evaluated by extensive simulations in different case studies from full orthogonalization to dynamic clustering and full reuse in the downlink and it is shown that proposed framework provides significant improvement over the benchmark cases.
\end{abstract}

Index Terms - backhauling, joint optimization, small cells, rate-balancing

\section{INTRODUCTION}

$\mathrm{C}$ apacity demand in cellular networks has been growing exponentially and this trend is expected to continue [1]. The ultimate vision is to provide consistent and reliable communication to create the perception of "infinite capacity" for end users. So far, Long Term Evolution of 3G (LTE) and LTE-Advanced [2]-[3] are key intermediate steps in smooth migration towards this vision of future wireless networks. In

This work was supported in part by the European Commission within the 7th Framework Program through the ICT Project iJOIN under Grant agreement 317941 and in part by the University of Surrey, Surrey, U.K., through the 5GIC programme.

The authors are with Institute for Communication Systems, home of the 5G Innovation Centre, Department of Electronic Engineering, University of Surrey, Guildford GU2 7XH, U.K (email: m.shariat@surrey.ac.uk) particular, small cells and advanced relay cells have emerged as fundamental elements in these systems [4]-[6] to enhance the efficiency in both capacity and coverage. On the other hand, a higher degree of interworking has been envisioned between small cells and conventional macro cells in the future via the notion of "dual connectivity", which implies that the user can have simultaneous connections to both macro and small cell stations [7]-[8]. This feature presents potential advantages such as separation between control and data channels where macro layer provides control signaling to small cells to enhance mobility, overhead and energy efficiency while small cells focus on information delivery in data plane [8]. This is the subject of an ongoing work in LTEAdvanced and beyond from Release 12 [7].

To support networks comprising several small cells, wired fiber backhauling might be cost-prohibitive or difficult to widely deploy in short to medium terms. Therefore, utilizing wireless backhauling seems a promising migration path. Emerging wireless systems have already taken initial steps in this direction, e.g. in 3GPP LTE-Advanced, layer 3 relaying strategies require self-backhauling on wireless LTE radio bearers to backhaul traffic between relay and donor eNodeBs [5]. Layer 3 relays mimic many of the functionalities in eNodeBs in smaller scale unlike conventional layer 1 or layer 2 relays.

Wireless backhauling for small cells and relay cells can introduce several challenges [6] when it comes to radio resource management (RRM) between the backhaul $(\mathrm{BH})$ and the access links. Here, coupling between the two can limit the multiuser diversity gain of radio resource allocation in the access link. In case of TDD between the transmission links, each packet would be received after two consecutive transmissions, i.e. on BH link to small cell station and from small cell station to the end user (access link). Therefore, the efficiency of system depends upon the balance of resources between the two links for each small cell. This requires efficient resource partitioning on $\mathrm{BH}$ or access links and ratebalancing strategies between the two.

\section{A. Related Works}

There exists a very large literature in the area of RRM and resource allocation for access radio networks like [9]-[10] where they fundamentally develop a framework for resource allocation in the context of orthogonal frequency division multiple access (OFDMA) systems. Also, there are several research studies addressing resource allocation strategies in 
the context of relay-based as well as small cell networks [11][18]. Here, [11] presents an integrated RRM solution for relaybased networks in the context of code division multiple access (CDMA) systems. Authors of [12]-[14] offer heuristic resource allocation strategies for in-band relaying in OFDMA networks. In particular, [14] focuses on uplink in-band relaying via co-scheduling and load balancing between macro and relay cell users. In [15], the authors explore resource allocation and relay selection strategies for OFDMA systems when a hybrid of amply-and-forward (AF) and decode-andforward (DF) relays, i.e. layer 1 and layer 2 relays are employed. In [16], the possibility of concurrent transmission between relays and macro cell networks is analyzed based on extreme value theory. Authors of [17] proposes a cooperative resource allocation strategy between macro and relay stations in downlink transmission and [18] focuses on cell-association strategies for small cells to achieve better load balancing between macro and small cells. A detailed survey on RRM for multi-carrier cellular networks can be found in [19]. Additionally, [20] provides an overview of radio resource allocation schemes designed for relay-enhanced systems.

The aforementioned research studies are quite inspiring. However, some are mainly focused on access side ([9]-[10] and [18]), in particular for conventional macro deployments ([9]-[10]). Others propose metrics that are not readily applicable to multi-carrier systems nor apply constraints imposed by BH limitation ([11], [17]). On the other hand, some solutions are heuristic in nature without detailed mathematical insights ([12]-[14]). More importantly, the majority of solutions assume a fixed duration of transmission between the $\mathrm{BH}$ and access links, not utilizing an important degree of freedom that we intend to explore in this paper.

\section{B. Contributions}

In this paper, we systematically decouple the problem of wireless backhauling for small cells via decomposition theory [21]-[22] taking into account the interaction of resource allocation parameters in different links and layers. Here, we extend our previous works in [23]-[24]. In [23], we proposed a low-complexity generic framework for resource allocation based on time-sharing applicable to different scenarios in downlink and uplink. On the other hand, in [24], we proposed an efficient graph-based dynamic clustering framework to control the level of co-tier interference between small cells. Combining the two solutions lead us to decouple the problem of resource allocation for small cells networks across $\mathrm{BH}$ and access links by introducing a set of governing variables as a priority factor. The decoupled elements facilitate efficient RRM strategies that are flexible and applicable to a wide set of cases from full orthogonalization to full spectrum reuse between small cells. In particular, we present a novel framework to update the duration of transmissions in downlink between the $\mathrm{BH}$ and access links in TDD mode in conjunction with resource allocation in both links. Here, the time coupling element across the links is challenging yet presents another degree of freedom to jointly optimize the system performance. The mathematical derivations and the proposed solutions are novel and have not been explored in the literature at this level to the best of our knowledge. Such RRM strategies enable flexible cloud-based radio access network (Cloud-RAN) that can be fully or partially driven by the Cloud [25]-[26]. The outcome solutions are evaluated by extensive simulations in different case studies for downlink transmission.

\section{SySTEM MODEL}

Here, the system consists of a local Cloud-RAN Gateway (L-GW) and a set of small cell stations $\boldsymbol{M}$, transmitting information to a set of users $\boldsymbol{N}$ over the set of sub-channels $\boldsymbol{K}$. The users are grouped into disjoint subsets $\boldsymbol{N}_{m}$ such that $\boldsymbol{N}=$ $\cup_{m}\left(\boldsymbol{N}_{m}\right)(m \in 1,2, \ldots, M)$. We also define $\boldsymbol{N}_{0}$ equivalent $\boldsymbol{M}$ to cover the $\mathrm{BH}$ transmission phase. We assume $l=1$ covers the $\mathrm{BH}$ transmission while $l=2$ refers to access link.

Figure 1 shows a typical example of our target network with $|M|=M=3,|N|=N=5$. Here, we assume that each sub-channel is constrained to be exclusively used by a single link within small cells per time instance. However, across different small cells, different case studies are considered from orthogonal allocation to dynamic clustering and finally the full reuse of sub-channels. The channel knowledge is considered to be available at L-GW entities connected to Cloud-RAN across the network. The Cloud-RAN processes the subchannel and power allocation and adjusts the transmission duration for $\mathrm{BH}$ and access links.

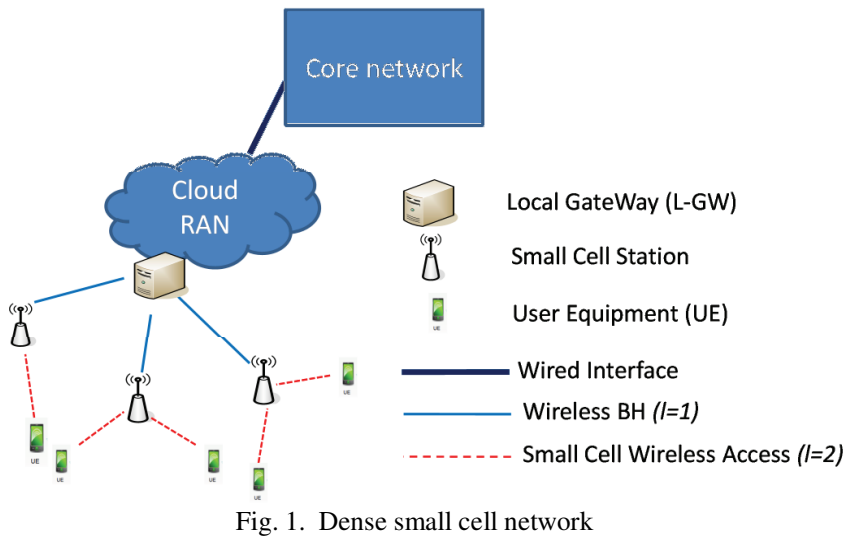

\section{PROBLEM FORMULATION}

The resource (sub-channel, power, transmission duration) allocation problem can be mathematically formulated as an optimization problem with a certain objective function subjects to involving constraints in the problem domain. Assuming weighted sum-rate (WSR) maximization as the optimization objective, a generic representation of the problem is as follows:

$$
\begin{aligned}
& \max _{\boldsymbol{p}, \boldsymbol{\tau}, \alpha} \sum_{n \in \boldsymbol{N}} w_{n} R_{n} \text { s.t. } \\
& \sum_{n \in N_{\widetilde{m}}} \sum_{k \in \boldsymbol{K}} \tau_{n, k}^{(l)} p_{n, k}^{(l)} \leq P_{\max , \widetilde{m}} \\
& \widetilde{m}=\left\{\begin{array}{cc}
0 & l=1 \\
m \in\{1,2, \ldots, M\} & l=2
\end{array}\right. \\
& \sum_{n \in N_{\widetilde{m}}} \tau_{n, k}^{(l)} \leq 1, \forall k \in K,
\end{aligned}
$$




$$
\begin{gathered}
\tau_{n, k}^{(l)} \in\{0,1\}, \quad \forall n \in N, \forall k \in K, \\
R_{n}=R_{n(m)}\left(\alpha, \tau_{n, k}^{(l)}, p_{n, k}^{(l)}\right), \quad m \in\{1,2, \ldots, M\} .
\end{gathered}
$$

Here, $R_{n}$ represents the outcome average rate of user $n$ over allocated sub-channels across $\mathrm{BH}$ and access links whereas $p_{n, k}^{(l)}$ is the instantaneous power that user $n$ transmits over subchannel $k$ on link $(l) . \tau_{n, k}^{(l)}$ denotes the fraction of time that user $n$ is allowed to exclusively use this sub-channel on link (l) per cell. Obviously, (2) provides the power limitation constraint per node, i.e. $P_{\max , \tilde{m}}$ whereas (3) in conjunction with (4) imposes the intra-cell orthogonality of resource allocation, i.e. the exclusivity constraint. Equation (5) shows that the rate $R_{n}$ per user $n$ would be equivalent to the rate allocated to this user out of its serving small cell, i.e. $m$. In particular, it is worth noting that $R_{n(m)}$ depends on the allocated share of resources $\tau_{n, k}^{(l)}, p_{n, k}^{(l)}$ to a small cell on different links in $\mathrm{BH}(l=1)$ and access sides $(l=2)$, the spectrum reuse strategy across different small cells and finally the parameter $\alpha$. Here, $\alpha \in(0,1)$ is the parameter to adjust the duration of transmission between $\mathrm{BH}$ and access links assuming TDD. We will detail this dependency later in the paper.

The grouping of (3) with (4) turns the optimization into a combinatorial problem that is intractable for large sets of subchannels and users. Furthermore, constraint (5) couples the resource allocation problem across both links and also between different small cells based on the spectrum reuse strategy.

In following sections, the joint resource allocation procedure is addressed in detail for different scenarios.

\section{A. Point-to-Point Small Cells (PPS)}

To simplify the analysis, first, a point-to-point model is considered where each small cell is exclusively associated to a single user.

Problem A. (PPS without time-sharing): Maximize the WSR of users by performing the resource allocation while satisfying the sub-channel and power allocation constraints.

This problem is very similar to the original problem (1)-(5). Here, we try to expand the definition of (5) from the capacity point of view taking into account PPS model:

$$
\begin{gathered}
R_{n}=R_{n(m)}\left(\alpha, \tau_{n, k}^{(l)}, p_{n, k}^{(l)}\right)=\min \left(\alpha r_{n}^{(1)},(1-\alpha) r_{n}^{(2)}\right) \\
r_{n}^{(l)}=\sum_{k \in \mathrm{K}} \tau_{n, k}^{(l)} \log _{2}\left(1+\rho_{n, k}^{(l)} p_{n, k}^{(l)}\right) .
\end{gathered}
$$

$r_{n}^{(l)}$ is the achievable rate of user $n$ at link $(l)$ whereas $\rho_{n, k}^{(l)}$ represents the SNR or SINR density function of the constituent link. In particular for the second phase, we have: $\rho_{n, k}^{(2)}=g_{n(m), k}^{(2)} /\left(\sum_{m^{\prime}} g_{n\left(m^{\prime}\right), k}^{(2)} p_{n\left(m^{\prime}\right), k}^{(2)}+\eta\right), m^{\prime} \neq m$.

Here, $g_{n(m), k}^{(2)}$ represents the channel gain of user $n$ from serving cell $m$ whereas $g_{n\left(m^{\prime}\right), k}^{(2)} p_{n\left(m^{\prime}\right), k}^{(2)}$ models the resulting interference from reusing the same sub-channel by small cell $m^{\prime}$ in non-orthogonal case studies. $\eta$ models the noise power over the target sub-channel. It is important to note that all the small cells are associated with L-GW of Cloud RAN for the BH link while each user is coupled to its corresponding small cell for the access link based on (7). As a result, we have: $p_{n, k}^{(1)}=p_{m, k}^{(1)}$ and $\rho_{n, k}^{(1)}=\rho_{m, k}^{(1)}$ where $m$ is the serving small cell for user $n$.

1) Relaxation

The problem A is still combinatorial due to the exclusivity constraint. Moreover, the minimization function as in (6) couples strongly the resource allocation across the links. To tackle these issues, the exclusivity constraint of (4) can be initially relaxed allowing the time-sharing of resources:

$$
\tau_{n, k}^{(l)} \in[0,1] \quad \forall n \in N, \forall k \in K .
$$

The exclusivity constraint can be re-imposed to the outcome solution, similar to the method we proposed in [23] with negligible performance loss. Additionally, the minimization problem can be interpreted as the following sub-problems:

Sub-problem A.1 (Bottleneck on the BH): In this regime, the achievable rate of access, from small cells to their associated users, is greater than the $\mathrm{BH}$. As a result, this subproblem is formulated as follows subject to the constraints (2), (3) and (8):

$$
\begin{gathered}
\max _{\boldsymbol{p}, \boldsymbol{\tau}, \alpha} \sum_{n \in \boldsymbol{N}} w_{n} \alpha r_{n}^{(1)} \text { s.t. } \\
\alpha r_{n}^{(1)} \leq(1-\alpha) r_{n}^{(2)}, \forall n \in N .
\end{gathered}
$$

As it can be seen, constraint (10) is employed to ensure the operation within the assumed regime.

Sub-problem A.2 (Bottleneck on the access): Here, contrary to the previous case, the $\mathrm{BH}$ is dominant due to the superiority of its channel condition. Hence, this sub-problem is formulated as follows subject to similar power and timesharing constraints as A.1:

$$
\begin{gathered}
\max _{\boldsymbol{p}, \boldsymbol{\tau}, \alpha} \sum_{n \in \boldsymbol{N}} w_{n}(1-\alpha) r_{n}^{(2)} \text { s.t. } \\
(1-\alpha) r_{n}^{(2)} \leq \alpha r_{n}^{(1)}, \forall n \in \boldsymbol{N} .
\end{gathered}
$$

Similar to sub-problem A.1, constraint (12) monitors the operation within the boundaries of the assumed regime. It can be easily shown that any other combination of rates can be mapped into one of the aforementioned regimes through rearranging the resources among the users. Hence, by assuming the operation in either regime, the relaxed problem can be efficiently solved.

Remark 1: Sub-problems A.1 and A.2 are not generally convex due to presence of inter-cell interference on the access links in case of spectrum reuse. However, by applying dynamic clustering framework proposed in [24], users of different cells can be effectively grouped into clusters with low-level of intra-cluster interference. Therefore, we decouple interference coordination problem from resource allocation by mapping the problem to this graph-based solution. This leads to near-convexity of the sub-problems above, enabling us to effectively decompose them as will be outlined below. 
To tackle the relaxed problems, we initially focus on A.1 and we form partial Lagrangian as below subject to constraints (2), (3) and (8):

$$
\begin{aligned}
& L(\boldsymbol{p}, \boldsymbol{\tau}, \alpha, \boldsymbol{\mu})=\sum_{n \in \boldsymbol{N}} w_{n} \alpha r_{n}^{(1)}- \\
& \sum_{n \in \boldsymbol{N}} \mu_{n}\left(\alpha r_{n}^{(1)}-(1-\alpha) r_{n}^{(2)}\right) .
\end{aligned}
$$

Here, the partial dual problem can be formed as $\xi(\boldsymbol{\mu}) \triangleq$ $\sup _{\boldsymbol{p}, \boldsymbol{\tau}, \alpha} L(\boldsymbol{p}, \boldsymbol{\tau}, \alpha, \boldsymbol{\mu})$ subject to time-share and power constraints where $\mu_{n}$ (or $\left.\boldsymbol{\mu}\right)$ are dual prices introducing a set of balancing parameters between $\mathrm{BH}$ and access links. As it can be seen, the sub-problem A.1 can be easily decoupled into two weighed sum-rate problems for both links based on (13) with weights $\phi_{n}^{(1)}=\left(w_{n}-\mu_{n}\right)$ and $\phi_{n}^{(2)}=\mu_{n}$, respectively. Similar decoupling can be achieved for A.2 by simply swapping the weights. This partial dual decomposition enables us to apply power and time-share allocation optimality conditions as derived in [23] with slight tuning of the weights for individual links.

2) Power allocation

Lemma 1: For a fixed time-sharing policy and transmission duration $\alpha$, the optimal power allocation variables for sub-problems A.1or A.2 will be the solutions of the following set of optimization problem:

$$
\begin{gathered}
p_{n, k}^{(l)}=\operatorname{argmax}_{\boldsymbol{p}}\left\{\phi_{n}^{(l)} \log _{2}\left(1+\rho_{n, k}^{(l)} p\right)-v_{m_{n}} p\right\}, \\
\forall n \in N, \forall k \in \boldsymbol{K},
\end{gathered}
$$

where $v_{m_{n}}$ are the power prices to satisfy (2) and $\phi_{n}^{(l)}$ are the balancing parameters to govern the balance between both links according to either (10) or (12).

Proof: It is straightforward to show (14) by applying standard dual decomposition with dual variables $v_{m_{n}}$ for (2) and considering $\mu_{n}$ as (13), where:

$$
\phi_{n}^{(l)}= \begin{cases}\left(w_{n}-\mu_{n}\right) & l=1 \\ \mu_{n} & l=2\end{cases}
$$

Following a similar approach for A.2, the same optimality condition holds unless (15) that should be amended as follows:

$$
\phi_{n}^{(l)}= \begin{cases}\mu_{n} & l=1 \\ \left(w_{n}-\mu_{n}\right) & l=2\end{cases}
$$

Corollary 1: The solution to the power allocation problems (14) is similar to the standard multi-level water-filling as in (17) where $[x]^{+}=\max (0, x)$.

$$
p_{n, k}^{(l)}=\left[\frac{\phi_{n}^{(l)}}{\ln (2) v_{m_{n}}}-\frac{1}{\rho_{n, k}^{(l)}}\right]^{+} .
$$

Proof: A straightforward result of Lemma 1.

3) Time-share allocation

Similar to power allocation scenario, by fixing the average powers $q_{n, k}^{(l)}=p_{n, k}^{(l)} \tau_{n, k}^{(l)}$ and $\alpha$, the time-share variables can be re-tuned to increase the aggregate WSR across the network.

Lemma 2: For fixed allocated average powers and transmission duration $\alpha$, the optimal time-share variables for sub-problems A.1 or A.2 will be the solutions of the following set of optimization problems:

$$
\begin{gathered}
\left(\tau_{1, k}^{(l)}, \ldots, \tau_{N, k}^{(l)}\right)=\operatorname{argmax}_{\tau}\left\{\sum_{n \in N} \phi_{n}^{(l)} \tau_{n} \log _{2}(1+\right. \\
\left.\left.\rho_{n, k}^{(l)} q_{n, k}^{(l)} / \tau_{n}\right)\right\}, \quad \forall k \in K,
\end{gathered}
$$

subject to the constraints (3) and (8).

Proof: By fixing the average allocated powers and the transmission duration, the optimization will be decomposed to the above equations per sub-channel as the power constraints (2) are the only factors that couple the optimization problem across the sub-channels [23].

Corollary 2: The time-share solutions of equation (18) can be found by solving the following set of equations:

$$
\begin{gathered}
U_{n}\left(y^{(l)}\right)=\phi_{n}^{(l)}\left[\log _{2}\left(1+y^{(l)}\right)-\frac{y^{(l)}}{\ln (2)\left(1+y^{(l)}\right)}\right]= \\
\lambda_{k}^{(l)}, y^{(l)}=\rho_{n, k}^{(l)} \frac{q_{n, k}^{(l)}}{\tau_{n, k}^{(l)}}
\end{gathered}
$$

where $U_{n}\left(y^{(l)}\right)$ is the marginal utility of user $n$ at link $(l)$ and $\lambda_{k}^{(l)}$ are the time-share prices to satisfy the constraints (3) and (8).

Proof: This is a direct result of Lemma 2 [23].

\section{4) Adjusting the duration of transmission}

Theorem 1: There is an optimum value of $\alpha$ where the solutions of both sub-problems A.1 and A.2 converge that is the optimal solution of problem A where:

$$
\frac{r_{n}^{*(2)}}{r_{n}^{*(1)}}=\frac{\alpha^{*}}{\left(1-\alpha^{*}\right)}, \forall n \in N, \alpha \in(0,1) .
$$

Proof: Considering the sub-problem A.1 and (13), the tuning parameters can be iteratively updated based on the subgradient method as follows:

$$
\begin{gathered}
\boldsymbol{\mu}^{(t+1)}= \\
{\left[\boldsymbol{\mu}^{(t)}+\varepsilon(t)\left(\alpha \boldsymbol{r}^{(1)(t+1)}-(1-\alpha) \boldsymbol{r}^{(2)(t+1)}\right)\right]_{D_{\boldsymbol{\mu}}}}
\end{gathered}
$$

where []$_{D_{\mu}}$ represents the projection of the dual variable on the corresponding domain $(\mu>0) . \varepsilon(t)$ is the coefficient that regulates the step-size in the (sub)gradient direction and can be chosen constant but sufficiently small to guarantee the convergence [21].

In similar manner for sub-problem A.2, the tuning parameters can be updated as follows:

$$
\begin{gathered}
\boldsymbol{\mu}^{(t+1)}= \\
{\left[\boldsymbol{\mu}^{(t)}+\varepsilon(t)\left((1-\alpha) \boldsymbol{r}^{(2)(t+1)}-\alpha \boldsymbol{r}^{(1)(t+1)}\right)\right]_{D_{\boldsymbol{\mu}}} .}
\end{gathered}
$$

As the dual problem converges to the optimal dual prices $\left(t \rightarrow \infty: \boldsymbol{\mu}^{(t+1)} \approx \boldsymbol{\mu}^{(t)}\right)$, we can clearly see that, there is a unique solution for $\alpha^{*}$ as below for both sub-problems:

$$
\text { Limit }_{t \rightarrow \infty} \frac{r_{n}^{(2)(t)}}{r_{n}^{(1)(t)}+r_{n}^{(2)(t)}}=\alpha^{*},
$$

$\forall n \in N$ where $\alpha \in(0,1)$. 
Considering the PPS model, each user is exclusively attached to a single small cell; therefore we can define $\alpha_{m}^{(t)}$ as below for each small cell per iteration:

$$
\alpha_{m}^{(t)}=\frac{r_{n}^{(2)(t)}}{r_{n}^{(1)(t)}+r_{n}^{(2)(t)}}
$$

$\forall n \in N, \forall m \in\{1,2, \ldots, M\}$.

Considering $\alpha_{m}^{(t)}$ as the optimal transmission duration for small cell $m$ at iteration $t$, the transmission durations might evolve independently for different cells across different iterations of the optimization process. However, they will converge to the optimal $\alpha^{*}$ asymptotically based on Theorem 1. Defining average $\bar{\alpha}$ as (25), it is straightforward to show that this average transmission duration value also converges asymptotically to optimal $\alpha^{*}$ based on (23) and (24).

$$
\bar{\alpha}^{(t)}=\frac{1}{M} \sum_{m} \alpha_{m}^{(t)}
$$

This averaging mechanism provides a unique value per iteration across all cells in order to update dual prices based on (23) or (24) that it asymptotically reaches optimal solution.

To understand the optimality condition, it is instructive to examine the effect of adjusting the transmission duration on the joint capacity of BH and access links:

By definition, the instantaneous capacity region $\boldsymbol{C}_{\boldsymbol{\pi}}(\boldsymbol{\rho})$ is a set that consists of all the achievable rate vectors for the current channel state vector $\boldsymbol{\rho}$ under the constraints of a given resource allocation policy $\boldsymbol{\pi}$. For example, in problem A, (2) provides the constraints on the power allocation $\boldsymbol{p}$ whereas (3) and (4) impose the constraints on the time-sharing policy $\boldsymbol{\tau}$. Mathematically, the instantaneous capacity region can be considered as the union of all achievable rate vectors under the considered policy:

$$
\boldsymbol{C}_{\boldsymbol{\pi}}(\boldsymbol{\rho})=\cup_{i} R_{i_{\pi}}(\boldsymbol{\rho}) \text { where } \boldsymbol{R}_{i_{\pi}}(\boldsymbol{\rho}) \text { satisfies } \boldsymbol{\pi} .
$$

In the case of joint $\mathrm{BH}$ and access links, each element of a typical achievable rate vector is the minimum of achievable rates (of a target user) on both links according to (6). Based on this assumption, for a fixed $\alpha$, the total capacity region will be the intersection of corresponding capacity regions on different links:

$$
\boldsymbol{C}_{\pi}(\alpha, \boldsymbol{\rho})=\boldsymbol{C}_{\pi}^{(1)}\left(\alpha, \boldsymbol{\rho}^{(\mathbf{1})}\right) \cap \boldsymbol{C}_{\pi}^{(2)}\left((1-\alpha), \boldsymbol{\rho}^{(\mathbf{2})}\right) .
$$

Here, increasing the transmission duration expands the capacity region of the BH while it will shrink the region on the access link and vice versa. The capacity region for any value of $\alpha$ can be considered as a typical set of achievable rate vectors. As a result, the union of these typical sets can form the total capacity region based on (26):

$$
\boldsymbol{C}_{\pi}(\boldsymbol{\rho})=\mathrm{U}_{\alpha} \boldsymbol{C}_{\pi}(\alpha, \boldsymbol{\rho})
$$

In the joint optimization problem (in either links) for a given weight vector, the optimal solution lies on the boundary of the capacity region to guarantee optimality. According to Theorem 1, there should be an optimal $\alpha$ where the solutions of both sub-problems converge. Intuitively, this unified solution should be located on the boundary of the capacity regions of both links. In other words, the boundaries of both links will intersect at the optimal $\alpha$. Consequently, the outcome boundary of the joint capacity region can be achieved by tracing intersection points for different values of $\alpha$. Figure 2 shows the boundaries of the capacity region across both links for a two-user scenario. As it can be seen, the intersection points of the corresponding boundaries (with the same values of $\alpha$ ) form the boundary of the joint capacity region in thick solid line. Figure 3 illustrates the capacity regions of the same scenario for a uniform quantized set of $\alpha$ (with 100 levels). As shown, the joint capacity region can be constructed as the union of capacity regions for different values of $\alpha$.

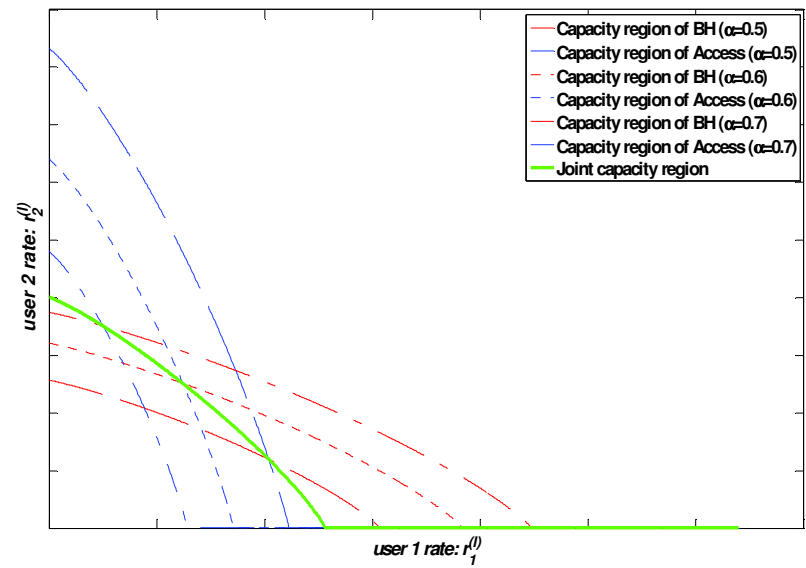

Fig. 2. The Capacity boundaries in joint $\mathrm{BH}$ and access links

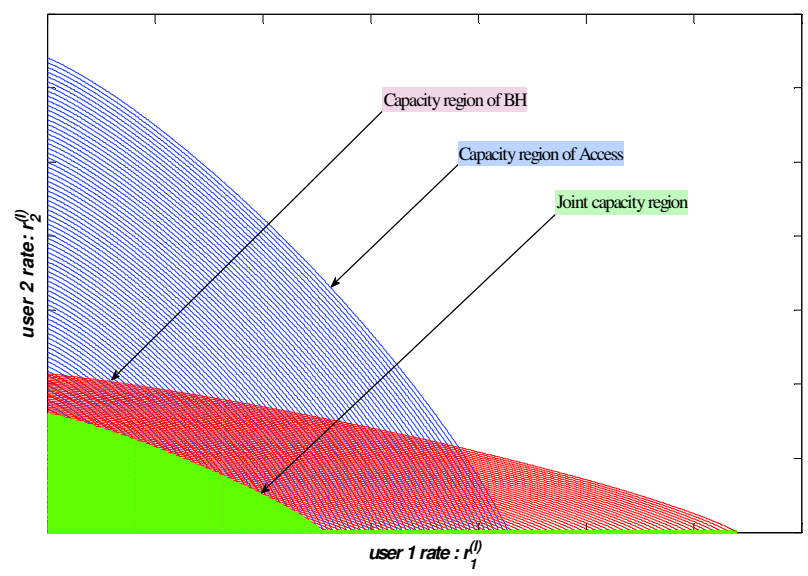

Fig. 3. The Construction of joint capacity region for small cells

\section{5) Sub-gradient method with small cell grouping}

As it was shown, the optimality conditions for both subproblems A.1 and A.2 follow a generic optimality conditions according to (17) and (19) per link where tuning parameter $\mu_{n}$ in conjunction with duration of transmission balance the operation across both links. In other words, the newly emerged tuning parameters act as a priority factor in the power and time-share allocation similar to weighting parameters $w_{n}$. As a result, there is a direct interaction between the resource allocation and rate-balancing between 
the $\mathrm{BH}$ and the access links. Therefore, each allocation policy introduces a new set of balancing parameters whereby each tuning set will directly affect the resource allocation.

Remark 2: In practice, as the resources are discrete (in the form of sub-channels), the resource allocation is quite sensitive to the tuning of balancing parameters. Therefore to come up with efficient and practical resource allocation strategies, some important observations should be taken into account:

- As the rate-balancing governs the coupling between the links, it requires a slower time-scale compared to the power and time-share update to ensure stability and convergence.

- The resource allocation is sensitive to the relative variations of tuning parameters rather than their absolute values.

The above remark leads us towards a coherent solution to update the tuning variables:

Small cell grouping (sub-algorithm): Per iteration of optimization, small cells can be classified based on satisfying or violating the balancing constraint that is associated with the problem. Considering the PPS model, each user is exclusively attached to a single small cell. Therefore, the constraints are equivalent to per user constraints. As an example, in subproblem A.1, users that satisfy the inequality (10) per iteration can be grouped as (over)satisfied users whereas others form the unsatisfied group. Intuitively, the tuning parameters of (over)satisfied users (or corresponding cells) can remain unchanged while the unsatisfied users are jointly updated with a similar increment. This method can avoid the unnecessary swapping of resources (sub-channels) inside (over)satisfied or unsatisfied groups and gradually increases the priority factor of the unsatisfied ones. As a result, the resources are more steadily transferred from the (over)satisfied group to unsatisfied one till the total balance is achieved for all the users (cells).

Based on this sub-algorithm, we can summarize the outcome joint resource allocation algorithm for problem $\mathrm{A}$ as below:

\section{Algorithm 1: Optimal Rate-Balancing}

1. Initialization: choose a sufficiently small uniform set for $\boldsymbol{\mu}^{(t=0)}$ and $\boldsymbol{\alpha}^{(t=0)}$.

2. Rate update: given $\boldsymbol{\mu}^{(t)}$, calculate $r^{(1)(t+1)}$ and $r^{(2)(t+1)}$ according to (17), (19) and (7).

3. Transmission duration update: update the transmission duration parameter according to (24) and (25).

4. Small cell grouping: classify the small cells (users) according to either (10) or (12).

5. Balancing update: update the balancing parameters $\boldsymbol{\mu}^{(t+1)}$ according to small cell grouping sub-algorithm.

6. Termination: stop when the total balance is achieved between all $\mathrm{BH}$ and access links otherwise go to 2 .

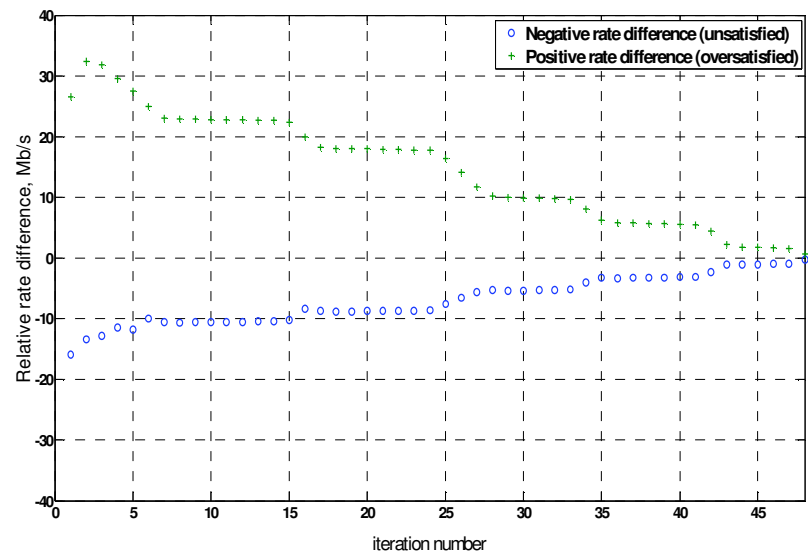

Fig. 4. Evolution of relative rate difference between access and BH links based on algorithm 1

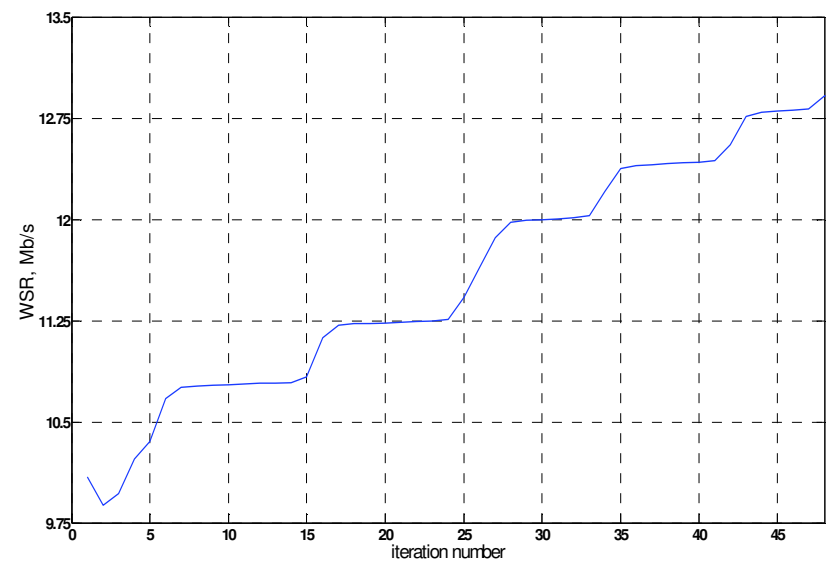

Fig. 5. Evolution of WSR in the network based on algorithm 1

Remark 3: In algorithm 1, the rate vectors of both links are updated based on the balancing parameters of the previous iteration. In practice, if the variations of balancing parameters are small compared to the weighting factors of users $w_{n}$, it is possible to fix the priority factor on the bottleneck link according to either (15) or (16). This approximation, even though sub-optimal simplifies the outcome algorithm by just tuning the rates on one link. This form is termed as suboptimal rate-balancing in the simulation section.

Figure 4 and Figure 5 illustrate a typical evolution of relative rate difference (imbalance) and WSR according to algorithm 1 in a two-user (two-cell) case. As shown, by each iteration, the sub-channels are transferred from the (over)satisfied user with positive rate difference to the unsatisfied one with negative rate difference and the transmission durations are adjusted accordingly. This procedure continues iteratively till the total balance is reached in both cells.

\section{B. Multi-access Small Cells (MAS)}

Here, the developed model of PPS is further extended for the MAS where each cell is associated with multiple users.

\section{1) Problem formulation}

In the case of MAS, the resource allocation in the BH link is not exclusive to one user per small cell. In other words, the 
achieved rate on the $\mathrm{BH}$ can be partitioned among all associated users of that small cell. To enable effective partitioning of resources in the $\mathrm{BH}$, we define new auxiliary variables $\tilde{r}_{n}^{(1)}$. Unlike $r_{n}^{(1)}$ in PPS case, $\tilde{r}_{n}^{(1)}$ are not directly defined based on the resource allocation policy as in (7) but they rather act as slicing variables to partition the resources allocated to a small cell station among its associated users [21]. This leads us to a new problem definition for MAS scenario:

Problem B. (MAS without time-sharing): This problem is similar to problem A. However, (7) should be defined separately for the $\mathrm{BH}$ and access links as below:

$$
\begin{gathered}
Q_{m}=\sum_{k \in \boldsymbol{K}} \tau_{m, k}^{(1)} \log _{2}\left(1+\rho_{m, k}^{(1)} p_{m, k}^{(1)}\right), \\
r_{n}^{(2)}=\sum_{k \in \boldsymbol{K}} \tau_{n, k}^{(2)} \log _{2}\left(1+\rho_{n, k}^{(2)} p_{n, k}^{(2)}\right) .
\end{gathered}
$$

Here, $Q_{m}$ represents the rate associated to the small cell $m$ in the $\mathrm{BH}$.

\section{2) Relaxation and decomposition}

Similar to the problem A, the exclusivity constraint can be relaxed through the time-sharing as in (8). Moreover, the minimization problem is decomposed into the following subproblems:

Sub-problem B.1 (Bottleneck on the BH): Similar to subproblem A.1 where (29)-(30) replace (7).

Sub-problem B.2 (Bottleneck on the access): Similar to sub-problem A.2 where (29)-(30) replace (7).

Please note auxiliary variables $\tilde{r}_{n}^{(1)}$ substitute $r_{n}^{(1)}$ in both sub-problems.

In sub-problem B.1, by forming the partial Lagrangian subject to the power and time-share constraints, we have:

$$
\begin{aligned}
& L(\boldsymbol{p}, \boldsymbol{\tau}, \alpha, \boldsymbol{\gamma})=\sum_{n \in \boldsymbol{N}} w_{n} \alpha \tilde{r}_{n}^{(1)}- \\
& \sum_{n \in \boldsymbol{N}} \gamma_{n}\left(\alpha \tilde{r}_{n}^{(1)}-(1-\alpha) r_{n}^{(2)}\right) .
\end{aligned}
$$

As mentioned, $\tilde{r}_{n}^{(1)}$ are not directly related to the resource allocation policy. As a result, algorithm 1 cannot be employed immediately based on sub-problem B.1.

3) Resource slicing

However, the following relationship should be satisfied between $Q_{m}$ and auxiliary variables $\tilde{r}_{n}^{(1)}$ as the feasibility constraint for the slicing variables:

$$
\sum_{n \in N_{m}} \tilde{r}_{n}^{(1)} \leq Q_{m}
$$

This implies that at the optimal point of operation with full balance, equality is achieved i.e. $\sum_{n \in N_{m}} \tilde{r}_{n}^{(1)}=Q_{m}$. Therefore, a new partial Lagrangian can be formulated taking into account the above as an additional constraint:

$$
\begin{gathered}
L(\boldsymbol{p}, \boldsymbol{\tau}, \alpha, \boldsymbol{\gamma}, \boldsymbol{\beta})=\sum_{n \in \boldsymbol{N}} w_{n} \alpha \tilde{r}_{n}^{(1)}- \\
\sum_{n \in \boldsymbol{N}} \gamma_{n}\left(\alpha \tilde{r}_{n}^{(1)}-(1-\alpha) r_{n}^{(2)}\right)- \\
\sum_{m \in \boldsymbol{M}} \beta_{m}\left(\sum_{n \in N_{m}} \tilde{r}_{n}^{(1)}-Q_{m}\right) .
\end{gathered}
$$

As it can be seen, two sets of price variables emerge in this sub-problem; $\gamma_{n}$ are price parameters to balance the achievable rate per user on access link to the sliced quota of the $\mathrm{BH}$, determined by $\tilde{r}_{n}^{(1)}$ whereas $\beta_{m}$ are dual variables in charge of matching the $\mathrm{BH}$ capacity to the aggregate sliced quota per small cell.

The dual form of sub-problem B.2 results in different price variables. In particular, this form can be more favorable due to direct relation between the access rates $r_{n}^{(2)}$ and the allocation policy according to (30) which is similar to (7) in problem A. Here, the equality relationship between $Q_{m}$ and the auxiliary variables $\tilde{r}_{n}^{(1)}$ at full balance can be utilized to better decouple the problem. Therefore, we can initially relax constraint (12) according to (32) to the following set of inequalities:

$$
\sum_{n \in N_{m}}(1-\alpha) r_{n}^{(2)} \leq \alpha Q_{m}, \forall m \in M
$$

As a result, the problem maps to the following partial Lagrangian subject to the power and time-share constraints:

$$
\begin{gathered}
L(\boldsymbol{p}, \boldsymbol{\tau}, \alpha, \boldsymbol{\theta})=\sum_{n \in \boldsymbol{N}} w_{n}(1-\alpha) r_{n}^{(2)}- \\
\sum_{m \in \boldsymbol{M}} \theta_{m}\left(\sum_{n \in \boldsymbol{N}_{m}}(1-\alpha) r_{n}^{(2)}-\alpha Q_{m}\right) .
\end{gathered}
$$

As it can be seen, the above closed form decouples the problem in the $\mathrm{BH}$ and access links. Here, Corollaries 1 and 2 can be efficiently utilized for the power and time-share allocation with the following slight modification:

$$
\phi_{n}^{(l)}= \begin{cases}\theta_{m} & l=1 \\ \left(w_{n}-\theta_{m}\right) & l=2\end{cases}
$$

where $\theta_{m}$ is the tuning set similar to $\mu_{n}$ that monitors the ratebalancing across the links. Unlike $\mu_{n}$, the new tuning set $\theta_{m}$ recovers the allocated rates to the $\mathrm{BH}$ of cells $Q_{m}$ rather than users on the first link. Rate-balancing algorithm (algorithm 1) can still be employed to calculate the optimal solution of problem B (via B.2) provided that $r_{1}^{(1)}$ and $r_{n}^{(2)}$ are substituted with $Q_{m}$ and $\sum_{n \in N_{m}} r_{n}^{(2)}$ respectively in steps 3 to 5 . Please note that auxiliary variables can be immediately recovered from (12) based on $r_{n}^{*(2)}$ and $\alpha^{*}$ assuming the equality holds. This would satisfy already relaxed constraint (12) and also (32).

\section{4) Complexity analysis}

Having the generic picture of the proposed algorithm based on MAS, here, we explore the complexity order of the algorithm. As a recap of notations, $N, K, M$ stand for the total number of users, sub-channels and small cells, respectively. We also assume that $T$ iterations will be required till the algorithm 1 converges. Each iteration of algorithm involves time share and power allocation updates according to (17) and (19). Assuming single iteration for time-share update and water-filing based power allocation, the complexity of these sub-problems will be $M K$ and $M K^{2}$ for the $\mathrm{BH}$ and $N K$ and $N K^{2}$ for access links. The duration of transmission update requires $M$ executions. Similarly, small cell grouping requires $M$ executions. There will be one iteration update for joint 
tuning of parameters $\theta_{m}$ according to the proposed algorithm. Therefore, summing up different steps, the whole complexity of the algorithm will be in the order of $\left[(M+N) K^{2}+\right.$ $(M+N) K+2 M+1] T$. We will provide more details on the convergence of algorithm and the required iterations $T$ for convergence in the simulation studies section.

\section{Special Case}

As a special case, a high capacity $\mathrm{BH}$ can be considered where a Line-of-Sight (LoS) symmetric connection is present between the L-GW of Cloud-RAN and its associated small cells. This assumption can simplify the rate-balancing procedure as we illustrate below in problem $\mathrm{C}$.

Problem C. (MAS with $\operatorname{LoS} B H)$ : Maximize the WSR on the access link subject to resource allocation constraints:

$$
\begin{gathered}
\max _{\mathbf{p}, \boldsymbol{\tau}, \alpha} \sum_{n \in N} w_{n}(1-\alpha) r_{n}^{(2)} \text { s.t. } \\
\sum_{n \in N}(1-\alpha) r_{n}^{(2)} \leq \alpha Q_{\max } .
\end{gathered}
$$

Here, $Q_{\max }$ shows the total achievable rate of the $\mathrm{BH}$ link that should be sliced among different small cells based on their access requirements.

Considering partial Lagrangian (subject to the resource allocation constraints), we have:

$$
\begin{gathered}
L(\boldsymbol{p}, \boldsymbol{\tau}, \alpha, \psi)=\sum_{n \in N^{W_{n}}}(1-\alpha) r_{n}^{(2)}- \\
\psi\left(\sum_{n \in \boldsymbol{N}}(1-\alpha) r_{n}^{(2)}-\alpha Q_{\max }\right) .
\end{gathered}
$$

As it can be seen, here, a single tuning parameter $\psi$ controls the balancing of rates among the small cells. Therefore, to meet the balance the transmission duration parameter can be directly tuned as follows:

$$
\alpha^{*}=\frac{\sum_{n \in N} r_{n}^{(2)}}{Q_{\max }+\sum_{n \in N} r_{n}^{(2)}}
$$

where $r_{n}^{(2)}$ can be directly calculated based on their respective weights of $w_{n} \cong\left(w_{n}-\psi\right)$ assuming $w_{n} \gg \psi$.

\section{Simulation STUdieS}

In this section, the developed concepts and algorithms are evaluated by means of extensive simulations.

\section{A. Case studies with orthogonal allocation between small cells}

Here, we consider topologies with orthogonal allocation between small cells. This enables us to focus on the efficiency proposed algorithms in different cases. We run Monte Carlo simulations based on the parameters consistent with 3GPP specifications [27] to examine the effect of bottleneck links on the considered algorithms. Furthermore, we assume normalized weights across the case studies here. Some common simulation parameters of case studies in A are presented in Table I.

\section{1) PPS Scenario}

Initially, we assume that PPS model is employed where each small is associated to a single user in its vicinity. The users are assumed to be almost stationary so the path loss and shadowing values are fixed during the simulation duration. The samples are averaged over 2000 independent snapshots. Furthermore, $\boldsymbol{\alpha}^{(t=0)}$ and $\boldsymbol{\mu}^{(t=0)}$ are set to 0.5 and 0.001 , respectively. For the symmetric (Sym) cases, the small cells are located at the perimeter of a circle with $200 \mathrm{~m}$ distance from the L-GW; Users are located at equal distance from their respective small cells $(500 \mathrm{~m})$. In asymmetric (Asym) scenarios, one small cell is shifted to the distance of $900 \mathrm{~m}$; however, the associated user keeps the same distance as before to it.

At first, a two-user case is considered under symmetric and asymmetric bottleneck conditions. Figure 6 shows the simulation result for this case. As it can be seen, ratebalancing provides considerable improvement in the efficiency of the resource allocation compared to the cases with independent resource allocation across $\mathrm{BH}$ and access links. In the case of symmetric bottleneck condition, the suboptimal algorithm provides near optimal performance whereas in asymmetric bottleneck condition, the gap to the optimal solution increases. This is consistent with our theoretical inspection in previous sections.

Figure 7 shows the simulation result for a three-user case. Here, similar trend is observable in both bottleneck conditions

Table I: Simulation parameters for case studies in A [27]
Small cell

\begin{tabular}{|c|}
\hline Small cell \\
\hline System bandwidth \\
\hline Carrier frequency \\
\hline Total TX power \\
\hline $\begin{array}{c}\text { Distance-dependent } \\
\text { path loss }\end{array}$ \\
Shadowing \\
\hline
\end{tabular}

Fast fading channel

UE / small cell dropping

$\mathrm{BH} \quad$ Access

\section{$5 \mathrm{MHz}$}

$2.0 \mathrm{GHz}$

$37 \mathrm{dBm}$

\begin{tabular}{c}
$\begin{array}{c}\text { Hotzone Model } 1 \\
\text { (Outdoor Macro) }\end{array}$ \\
$\begin{array}{c}\text { Hotzone Model } 1 \\
\text { (Outdoor Pico) }\end{array}$ \\
\hline $\begin{array}{c}\text { Lognormal, zero mean, } 8 \mathrm{~dB} \text { standard } \\
\text { deviation }\end{array}$ \\
\hline Rayleigh block fading \\
Variable based on scenario
\end{tabular}

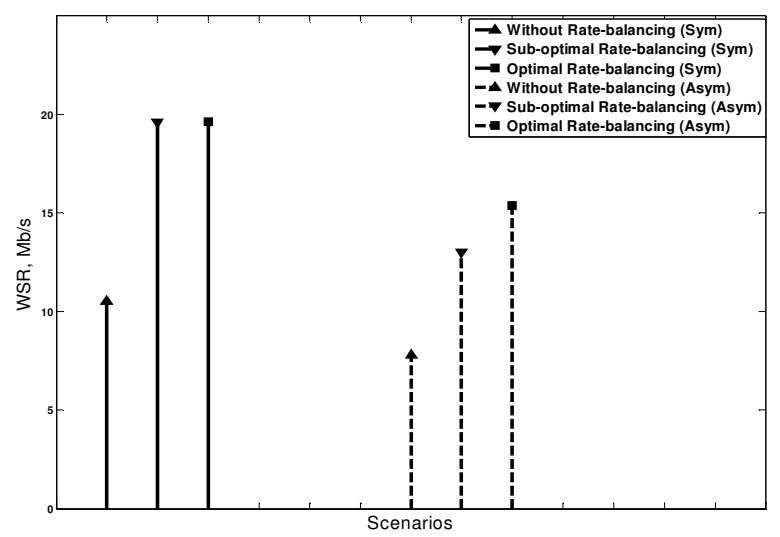

Fig. 6. Evaluating algorithmic efficiency of rate-balancing for A-1 $(M=2)$ 


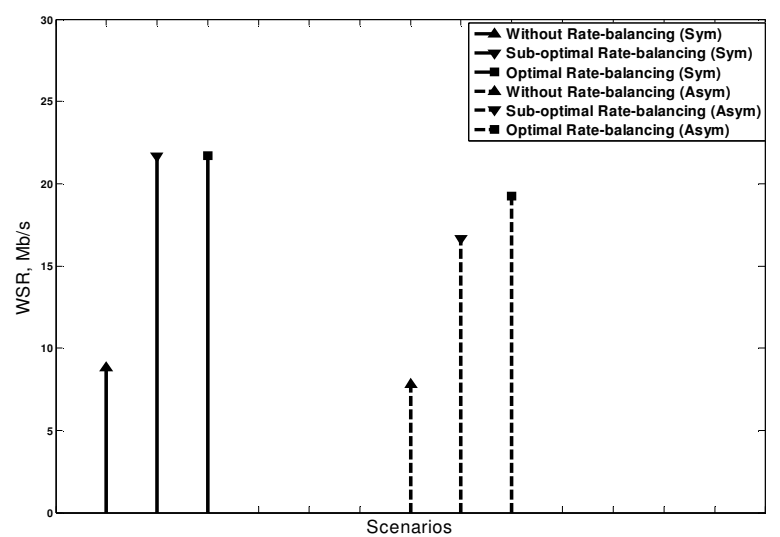

Fig. 7. Evaluating algorithmic efficiency of rate-balancing for A-1 $(M=3)$

\section{2) MAS Scenario}

In second scenario, the MAS model is utilized where each small cell is associated with multiple users. Here, the users are assumed to be mobile. As a result, the shadowing and path loss values changes across different snapshots. The samples are averaged over 2000 independent snapshots. $\boldsymbol{\alpha}^{(t=0)}$ and $\boldsymbol{\mu}^{(t=0)}$ are set as PPS case. The small cells are located at the perimeter of a circle with $500 \mathrm{~m}$ distance from the L-GW and the users are randomly but uniformly distributed in the vicinity of each small cell with a similar maximum distance from their respective small cell station.

Figure 8 shows the simulation result for different numbers of users in this scenario $(M=2)$. As expected, increasing the number of users enhances the WSR in all cases due to the better multiuser diversity. Rate-balancing provides significant improvement in the efficiency of resource allocation compared to the benchmark algorithms. This scenario indicates that in a realistic case where a mixture of symmetric and asymmetric bottleneck conditions exists, the performance of the suboptimal algorithm is quite close to the optimal solution. As a result, the sub-optimal algorithm can be considered as an alternative to the optimal one.

\section{B. Case studies with spectrum reuse between small cells}

In these case studies, we evaluate the performance of the proposed algorithm in a more detailed system-level topology again consistent with 3GPP specifications [27]. In particular, we examine the impact of different interference coordination strategies from dynamic clustering to full reuse. The common simulation parameters of case studies in B are presented in Table II. Here, we have an outdoor random cell and user deployment in a cluster in line with scenario 2 of [28]. In particular, four small cell stations are randomly dropped in a cluster area (ring) with minimum distances as outlined in Table II. Concerning the intra-cell scheduling, proportional fair (PF) scheduling is used for a multi-channel system per small cell to provide a fair allocation of resources between multiple users. Therefore, user weights are tuned based on this algorithm and are normalized to total weights per cell for a fair comparison. The samples are averaged over 5000 independent snapshots. Furthermore, $\boldsymbol{\alpha}^{(t=0)}$ and $\boldsymbol{\mu}^{(t=0)}$ are set as case studies in A.

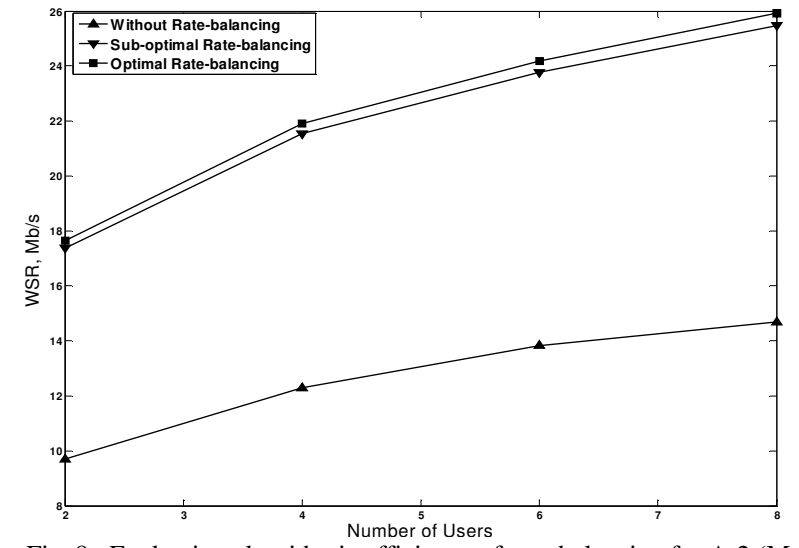

Fig. 8. Evaluating algorithmic efficiency of rate-balancing for A-2 (M=2)

Table II. Simulation parameters for case studies in B [27] Small cell $\mathrm{BH}$ $10 \mathrm{MHz}$

System bandwidth

Carrier frequency $2.0 \mathrm{GHz}$

Total TX power $24 \mathrm{dBm}$

\begin{tabular}{|c|c|c|}
\hline Distance-dependent path loss & $\begin{array}{l}\text { Macro-to-Relay } \\
\text { (Outdoor) }\end{array}$ & $\begin{array}{l}\text { Relay-to-UE } \\
\text { (Outdoor) }\end{array}$ \\
\hline Shadowing & \multicolumn{2}{|c|}{$\begin{array}{l}\text { Lognormal, zero mean, } 10 \mathrm{~dB} \\
\text { standard deviation }\end{array}$} \\
\hline $\begin{array}{l}\text { Antenna gain / connector } \\
\text { loss }\end{array}$ & \multicolumn{2}{|c|}{$5 \mathrm{dBi}$ (omni) / $0 \mathrm{dBi}$} \\
\hline Fast fading channel & \multicolumn{2}{|c|}{ Rayleigh block fading } \\
\hline UE dropping & \multicolumn{2}{|c|}{8 users per cell (32 in total) } \\
\hline $\begin{array}{l}\text { Radius for small cell } \\
\text { dropping in a cluster }\end{array}$ & \multicolumn{2}{|c|}{$90 \mathrm{~m}$} \\
\hline $\begin{array}{l}\text { Minimum small cell station } \\
\text { to UE distance }\end{array}$ & \multicolumn{2}{|c|}{$5 \mathrm{~m}$} \\
\hline $\begin{array}{l}\text { Minimum small cell station } \\
\text { to base station distance }\end{array}$ & \multicolumn{2}{|c|}{$20 \mathrm{~m}$} \\
\hline
\end{tabular}

1) Dynamic clustering

The proposed solution is initially studied under the dynamic clustering as interference coordination [24]. Here, we compare three RRM schemes:

- Without rate-balancing, where independent resource scheduling happens on the $\mathrm{BH}$ and access links

- Fixed partitioning, where the BH resource are equally partitioned between the small cells in a static manner,

- Rate-balancing, where we employ the proposed scheme combined with dynamic clustering.

We consider two performance indicators, CDF of normalized WSR (per cell) and CDF of user spectral efficiency in $(\mathrm{b} / \mathrm{s} / \mathrm{Hz})$.

As it can be seen in Figure 9 and Figure 10, the proposed rate-balancing scheme can provide significant improvement in both user spectral efficiency and WSR (per cell) in this case study for both performance indicators. It is also interesting to note that fixed partitioning on the $\mathrm{BH}$ can be generally a better strategy compared to independent but dynamic RRM in $\mathrm{BH}$ and access links considering the results without ratebalancing. However, fixed partitioning can be an expensive solution for small cell operation as it yields low BH utilization when it comes to small cells in low-load scenarios. 


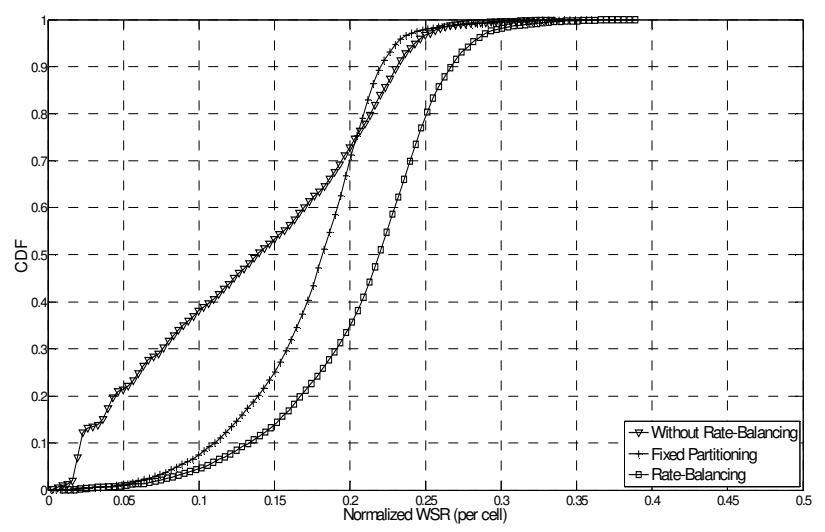

Fig. 9. CDF of normalized WSR for case study B-1

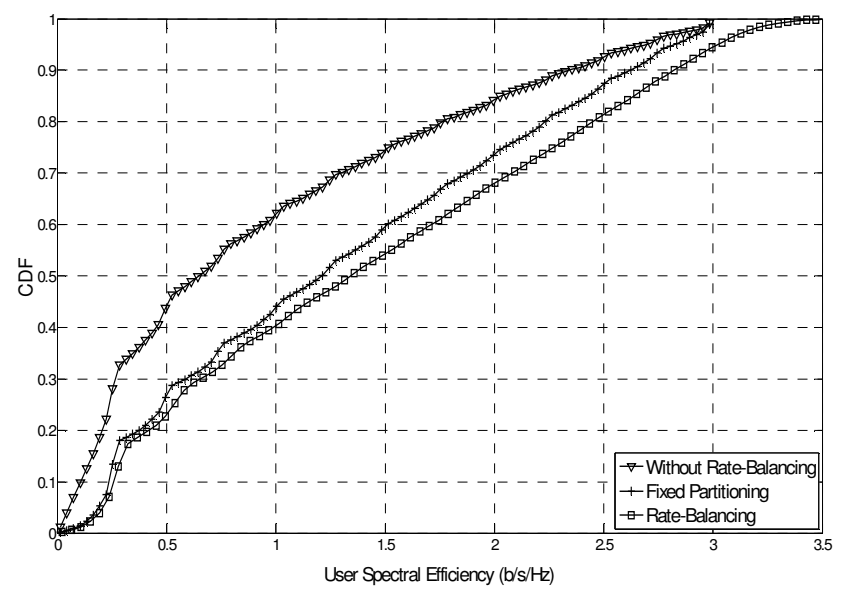

Fig. 10. CDF of user spectral efficiency for B-1

\section{2) Reuse one}

At this case study, we apply the rate-balancing mechanism in reuse one without any active interference coordination. The results are compared for similar RRM strategies and performance indicators as B-1.

Figure 11 and Figure 12 show simulations results of this case study. As it can be seen, similar trend is observable as B1 for the performance indicators in particular for CDF of WSR (per cell). It is worth noting that in reuse one case, the optimization problem and related capacity regions on $\mathrm{BH}$ and access links are not generally convex. As a result, the duality gap of proposed solution will be non-zero. Nevertheless, the system performance can still benefit from rate-balancing and the joint optimization as it is evident by the results. However, the major gain of the algorithm is achieved when ratebalancing strategies are employed in conjunction with interference coordination algorithms like dynamic clustering.

3) Analysis on evolution of $\alpha$ and convergence rate

In this sub-section, we provide more detailed analysis on evolution of $\alpha$ as the adjusting parameter for the duration of phases in the dynamic clustering case. Furthermore, we examine the convergence rate of the algorithm across different snapshots for the same case.

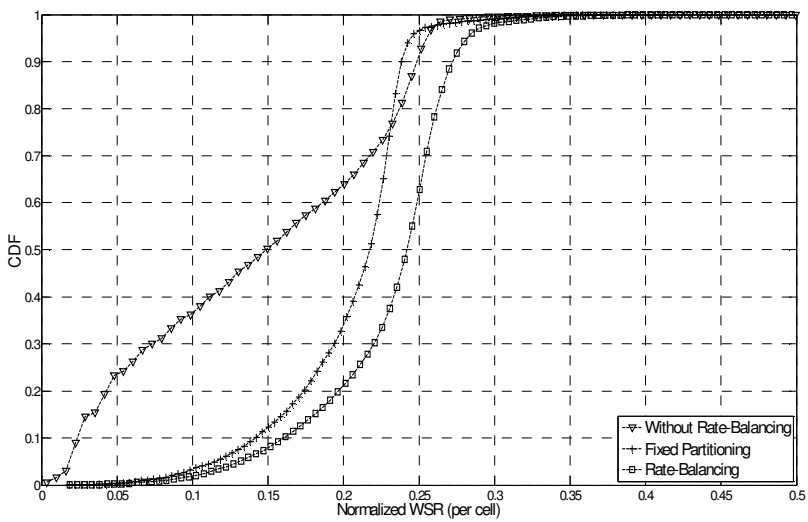

Fig. 11. CDF of normalized WSR for case study B-2

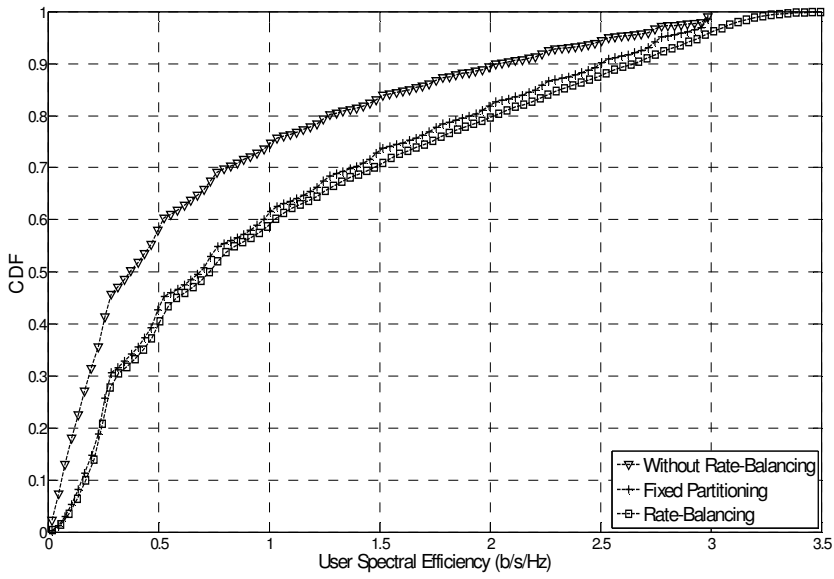

Fig. 12. CDF of user spectral efficiency for B-2

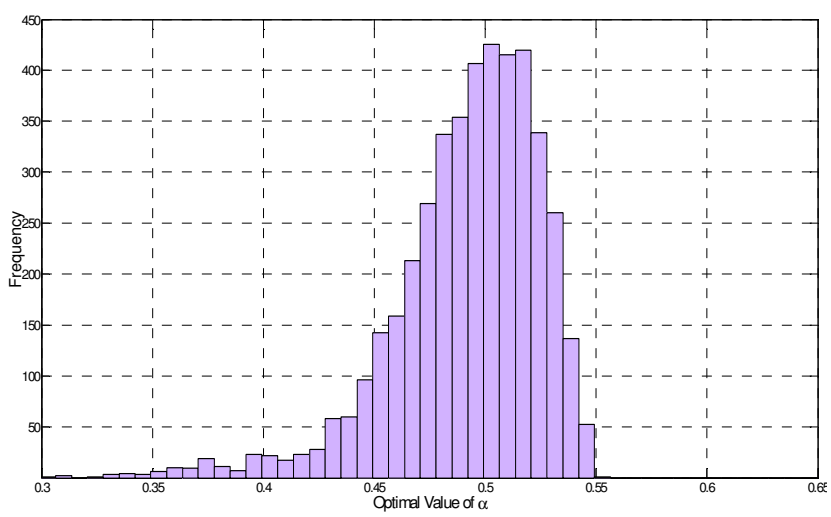

Fig. 13. Histogram of optimal value of $\alpha$

Figure 13 shows the histogram of optimal value of $\alpha$ across different snapshots. As it can be seen, the value is dynamically adjusted based on the instantaneous channel conditions in both links across different snapshots.

Figure 14 illustrates the histogram of the required number of iterations (for convergence) across different snapshots. As shown, the algorithm can converge in all snapshots with fewer than 250 iterations for $T$. Specifically, the majority of snapshots will converge in around 100 iterations. 


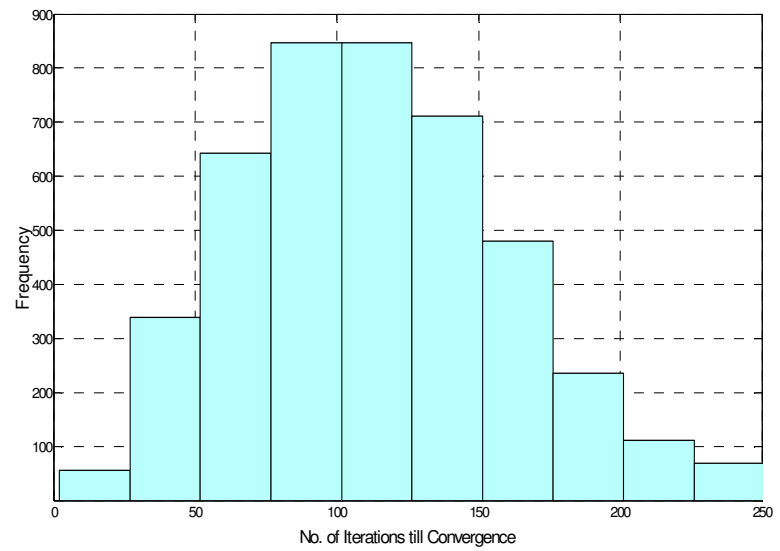

Fig. 14. Histogram of required number of iterations

\section{CONCLUSIONS}

In this paper, the problem of joint resource allocation between $\mathrm{BH}$ and access links in TDD was addressed for dense small cell networks. The problem was mathematically decomposed into per link sub-channel and power allocation where a set of rate-balancing parameters combined with phase duration parameter governed the coupling among the links. Moreover, novel algorithms were derived for rate-balancing by employing the concepts of small cell grouping and resource slicing. Finally, the efficiencies of proposed concepts and algorithms were evaluated by system-level simulations. As shown, joint optimization with rate-balancing could provide significant improvement over independent resource allocation across $\mathrm{BH}$ and access links and also fixed $\mathrm{BH}$ partitioning strategies. In particular, the proposed algorithm could deliver significant performance improvement in conjunction with efficient interference coordination strategies like dynamic clustering.

\section{ACKNOWLEDGMENT}

The views and conclusions contained here are those of the authors and should not be interpreted as necessarily representing the official policies or endorsements, either expressed or implied, of the iJOIN project or the European Commission.

\section{REFERENCES}

[1] Requirements, evaluation criteria, and submission templates for the development of IMT-Advanced, ITU-R, Accessed May 2014, [Online] Available: http://www.itu.int/md/R07-SG05-C-0068/

[2] Evolved Universal Terrestrial Radio Access (E-UTRA) and Evolved Universal Terrestrial Radio Access Network (E-UTRAN), Overall Description, 3GPP TS 36.300, Mar. 2014

[3] Further Advancements for E-UTRA Physical Layer Aspects, 3GPP TR 36.814, Mar. 2010

[4] A. Damnjanovic, J. Montojo, Yongbin Wei, Tingfang Ji, Tao Luo, M. Vajapeyam, Taesang Yoo, Osok Song, and D. Malladi, "A survey on 3GPP heterogeneous networks," Wireless Commun., vol.18, no.3, pp.1021, Jun. 2011

[5] H. Raza, "A brief survey of radio access network backhaul evolution: part II,” IEEE Commun. Mag., vol.51, no.5, pp.170-177, May 2013

[6] C. Hoymann, Wanshi Chen, J. Montojo, A. Golitschek, C. Koutsimanis, and Xiaodong Shen, "Relaying operation in 3GPP LTE: challenges and solutions,” IEEE Commun. Mag.,vol.50, no.2, pp.156-162, Feb. 2012
[7] C. Hoymann, D. Larsson, H. Koorapaty and Jung-Fu Cheng, "A Lean Carrier for LTE,” IEEE Commun. Mag., vol.51, no.2, pp.74-80, Feb. 2013

[8] H. Ishii, Y. Kishiyama, and H. Takahashi, "A novel architecture for LTE-B :C-plane/U-plane split and Phantom Cell concept," in Proc. IEEE Globecom Workshops, 2012, pp.624-630

[9] G. Song and Ye Li, "Cross-layer optimization for OFDM wireless networks-part I: theoretical framework," IEEE Trans. Wireless Commun., vol.4, no.2, pp.614-624, Mar. 2005

[10] G. Song and Ye Li, "Cross-layer optimization for OFDM wireless networks-part II: algorithm development," IEEE Trans. Wireless Commun., vol.4, no.2, pp.625-634, Mar. 2005

[11] Y. Liu, R. Hoshyar, X. Yang, and R. Tafazolli, "Integrated Radio Resource Allocation for Multihop Cellular Networks With Fixed Relay Stations," IEEE J. Sel. Areas Commun., vol.24, no.11, pp.2137-2146, Nov. 2006

[12] Lei Huang, Mengtian Rong, Lan Wang, Yisheng Xue, and E. Schulz, "Resource Allocation for OFDMA Based Relay Enhanced Cellular Networks," in Proc. IEEE VTC, 2007, pp.3160-3164

[13] Zhuyan Zhao, Jian Wang, S. Redana, and B. Raaf, "Downlink Resource Allocation for LTE-Advanced Networks with Type1 Relay Nodes," in Proc. IEEE VTC, 2012, pp.1-5

[14] K. Dereje Woldemedhin, O. Bulakci, A. B. Saleh, S. Redana, and F. Granelli, "Joint backhaul co-scheduling and relay cell extension in LTEadvanced networks uplink performance evaluation," in Proc. European Wireless, 2012, pp.1-8

[15] T.C.Y. Ng and Wei Yu, "Joint optimization of relay strategies and resource allocations in cooperative cellular networks," IEEE J. Sel. Areas Commun., vol.25, no.2, pp.328-339, Feb. 2007

[16] O. Oyman, "Opportunistic scheduling and spectrum reuse in relay-based cellular networks," IEEE Trans. Wireless Commun., vol. 9, no. 3, pp. 1074-1085, Mar. 2010

[17] Qian Li, R.Q. Hu, Yi Qian, and Geng Wu, "Intracell Cooperation and Resource Allocation in a Heterogeneous Network with Relays," IEEE Trans. Veh. Technol., vol.62, no.4, pp.1770-1784, May 2013

[18] R. Madan; J. Borran, A. Sampath, N. Bhushan, A. Khandekar, and Tingfang $\mathrm{Ji}$, "Cell Association and Interference Coordination in Heterogeneous LTE-A Cellular Networks," IEEE J. Sel. Areas Commun., vol.28, no.9, pp.1479-1489, Dec. 2010

[19] S. Sadr, A. Anpalagan, and K. Raahemifar, "Radio Resource Allocation Algorithms for the Downlink of Multiuser OFDM Communication Systems," Commun. Surveys Tuts., vol.11, no.3, pp.92-106, 3rd quarter 2009

[20] M. Salem, A. Adinoyi, M. Rahman, H. Yanikomeroglu, D. Falconer, Y.D. Kim, and Y.-C. Cheong, "An overview of radio resource management in relay-enhanced OFDMA-based networks," Commun. Surveys Tuts., vol.12, no.3, pp.422-438, 3rd quarter 2010

[21] D.P. Palomar and Mung Chiang, "A tutorial on decomposition methods for network utility maximization," IEEE J. Sel. Areas Commun., vol.24, no.8, pp.1439-1451, 2006

[22] D.P. Palomar and Mung Chiang, "Alternative Distributed Algorithms for Network Utility Maximization: Framework and Applications," IEEE Trans. Autom. Control, vol.52, no.12, pp.2254-2269, Dec. 2007

[23] R. Hoshyar, M. Shariat, and R. Tafazolli, "Subcarrier and Power Allocation with Multiple Power Constraints in OFDMA Systems," IEEE Commun. Lett., vol.14, no.7, pp. 644-646, Jul. 2010

[24] E. Pateromichelakis, M. Shariat, A. Quddus, M. Dianati, and R. Tafazolli, "Dynamic Clustering Framework for Multi-Cell Scheduling in Dense Small Cell Networks," IEEE Commun. Lett., vol.17, no.9, pp.1802-1805, Sep. 2013

[25] EU FP7 project iJOIN, "iJOIN I Interworking and JOINt Design of an Open Access and Backhaul Network Architecture for Small Cells based on Cloud Networks," Accessed May 2014, [Online] Available: http://www.ict-ijoin.eu

[26] P. Rost, C. J. Bernados, A. De Domenico, M. Di Girolamo, M. Lalam, A. Maeder, D. Sabella, and D. Wubben, "Cloud Technologies for Flexible 5G Radio Access Networks," IEEE Commun. Mag., vol.52, no.5, May 2014

[27] Evolved Universal Terrestrial Radio Access (E-UTRA): Further advancements for E-UTRA physical layer aspects, 3GPP TR 36.814, Mar. 2010

[28] Small cell enhancements for E-UTRA and E-UTRAN-Physical layer aspects, 3GPP TR 36. 872, Dec. 2013 